\title{
Mas allá de los nudges: Políticas públicas efectivas basadas en la evidencia de las ciencias del comportamiento
}

\section{Beyond Nudges: Behavioral Based Evidence Based Public Policies}

\author{
Antonio Cabrales Goitia \\ Universidad Carlos III de Madrid (España) \\ ORCID: https://orcid.org/0000-0003-0209-0399 \\ antonio.cabrales@uc3m.es
}

\section{NOTA BIOGRÁFICA}

Antonio Cabrales es doctor en Economía por UCSD y catedrático de la Universidad Carlos III. Ha sido catedrático en el University College London y en la Universidad Pompeu Fabra. Es vicepresidente ejecutivo de la EEA. Trabaja en teoría de juegos, y economía experimental. Es editor asociado del Journal of Economic Theory. Ha publicado en revistas como AER, JPE, REStud, RFS, MS y PRL.

Pedro Rey Biel

ESADE-Universtat Ramón Llull (España) ORCID: https://orcid.org/0000-0002-1606-2631

\section{NOTA BIOGRÁFICA}

Pedro Rey Biel es Profesor Titular de Economía del Comportamiento en el Departamento de Economía, Finanzas y Contabilidad de ESADE y Director del Policy Impact Lab de ESADE EcPol. Máster en Economía y Finanzas por el CEMFI y Doctor en Economía por University College London.

\section{RESUMEN}

Los nudges son un mecanismo psicológico que puede inducir cambios de comportamiento en los individuos a bajo coste y, por ello, se han convertido en un instrumento de política pública que ha demostrado tener éxito en algunos contextos y que cada vez es más aceptado. Sin embargo, para dirigir la modernización de la administración pública hacia el uso de políticas públicas basadas en la evidencia se debe abrazar de forma más ambiciosa el enfoque de la Economía del Comportamiento y no limitarse al uso de nudges, sino incluir otros instrumentos complementarios y sustitutivos, como los incentivos, que tengan en cuenta la diversidad en los procesos de toma de decisiones de los individuos y el amplio espectro de motivaciones que las generan. La experimentación, la disponibilidad de datos públicos, y la evaluación de las políticas públicas son pasos que deben acompañar a la adopción de la economía del comportamiento por parte de la administración pública en España.

\section{PALABRAS CLAVE}

Economía del comportamiento; políticas basadas en la evidencia; nudges; incentivos; paternalismo libertario; manipulación; experimentación; sesgos de comportamiento. 
GAPP. Nueva Época - N. 25, marzo 2021 - ISSN: 1989-8991 - DOI: https://doi.org/10.24965/gapp.i25.10864 - [Págs. 38-45] Número monográfico - Los nudges y el diseño conductual de políticas públicas

Mas allá de los nudges: Políticas públicas efectivas basadas en la evidencia de las ciencias del comportamiento

Antonio Cabrales / Pedro Rey Biel

\begin{abstract}
Nudges are one of the most accepted public policy instruments, due to the success of some recent interventions and its relative low implementation cost. Nevertheless, in order to achieve the modernization of our public administration towards evidence based policies, we should embrace Behavioral Economics in a more ambitious manner, not limiting ourselves to nudges, but including other instruments, such as incentives designed taking into account the wide array of human motivations and the array of individual decision making processes. Experimentation, public access to administrative data, and policy evaluation are steps that will go hand in hand with the adoption of behavioral economics by the Spanish public administration.
\end{abstract}

\title{
KEYWORDS
}

Behavioral economics; evidence based policies; nudges; incentives; libertarian paternalism; manipulation; experimentation; behavioral biases.

\section{SUMARIO}

1. INTRODUCCIÓN. 2. INTERVENCIONES BASADAS EN LA ECONOMÍA DEL COMPORTAMIENTO: NUDGES VS INCENTIVOS. 3. EL AUGE DE LOS NUDGES COMO INTRUMENTO DE POLÍTICA PÚBLICA. 4. RESERVAS AL USO DE LOS NUDGES. 5. CONCLUSIONES Y RECOMENDACIONES. 6. REFERENCIAS BIBLIOGRÁFICAS.

\section{INTRODUCCIÓN ${ }^{1}$}

Las políticas públicas son el sistema de leyes, medidas reguladoras y otras acciones que los gobiernos diseñan e implementan en respuesta a los problemas de sus ciudadanos, buscando mejorar su bienestar. En cierta forma, el objetivo de las políticas públicas puede ser definido como el de reducir las decisiones subóptimas por parte de los individuos y corregir los posibles efectos negativos de estas decisiones para la sociedad. No obstante, el éxito de las políticas públicas depende crucialmente de la respuesta de los ciudadanos ante ellas. Es decir, de la aceptación, comprensión y grado de cumplimiento que hacen los ciudadanos de las medidas que se les aplican. Sin embargo, en contra de los postulados tradicionales de la Economía, que en el fondo es una de las ciencias sociales que estudia cómo se toman decisiones cuando éstas tienen consecuencias, existe amplia evidencia de que los seres humanos no somos racionales y, por tanto, nuestras decisiones frecuentemente no redundan en nuestro propio beneficio o en el de la sociedad a la que pertenecemos.

Ya desde la Teoría de los Sentimientos Morales de Adam Smith (1759) y la Introducción a los Principios Morales y Legislativos de Jeremy Bentham (1789), la racionalidad en economía se define como el proceso que lleva a tomar decisiones optimizadoras que redundan en el propio beneficio. Esto implica que, a la hora de elegir entre diversas opciones, los individuos son capaces de valorar correctamente el beneficio (la «utilidad») que deriva de las consecuencias de cada una de sus opciones y siempre eligen aquella que les reporta más satisfacción.

En los últimos cincuenta años, la Economía del Comportamiento ha demostrado que muchas de las acciones de los seres humanos no siempre están regidas por el principio de racionalidad, sino que, en muchas ocasiones cometemos errores respecto a las acciones que maximizan nuestro bienestar. Como indican Kahneman, Slovic y Tversky (1974), nuestro proceso de toma de decisiones frecuentemente no consiste en un cálculo frío valorando alternativas, sino que se basa em decisiones automáticas, no necesariamente meditadas (sesgos o heuristics), influidas a su vez por nuestras emociones, nuestra incapacidad de procesar la información, nuestro comportamiento ante la incertidumbre o simplemente, porque no sabemos exactamente qué es lo que más nos conviene, ya sea en el corto o en el largo plazo. La Economía del comportamiento ha basado, por tanto, parte de su éxito en tomar como punto de partida lo que hacen los individuos realmente, en lugar de una idealización «teórica» y simplificada sobre cómo se comportan (o deberían comportarse), para, a partir de ahí, estudiar el proceso de toma de decisiones como un proceso potencialmente subóptimo, en el que la influencia de los sesgos psicológicos puede ser importante.

\footnotetext{
1 Los autores agradecen a los editores y evaluadores sus comentarios a una versión previa de este documento.
} 
GAPP. Nueva Época - N. ${ }^{\circ}$ 25, marzo 2021 - ISSN: 1989-8991 - DOI: https://doi.org/10.24965/gapp.i25.10864 - [Págs. 38-45] Número monográfico - Los nudges y el diseño conductual de políticas públicas

Mas allá de los nudges: Políticas públicas efectivas basadas en la evidencia de las ciencias del comportamiento

Teniendo en cuenta las enseñanzas de la Economía del Comportamiento (Behavioral Economics), la dificultad a la que se enfrenta el éxito de las políticas públicas es doble. No sólo deben superar al individuo proporcionándole un contexto que maximice su bienestar, incluso cuando esos mismos individuos pueden desconocer ese mismo bienestar, sino que deben anticipar cómo reaccionarán los individuos ante ese contexto para que sus acciones no descabalguen el propósito con el que fueron diseñadas.

El movimiento Nudge (disculpen el anglicismo pero no nos terminan de convencer términos como «acicate» o, el aún más tierno, «empujoncito»), liderado por el influyente libro de Thaler y Sunstein (2009), supone un instrumento especialmente atractivo para quienes diseñan e implementan políticas públicas: por una parte, los nudges asumen qué es lo mejor para el individuo o para la sociedad, impone una función objetivo sin necesidad de que sus integrantes se la cuestionen y, si son efectivos, consiguen que los ciudadanos se comporten como se espera de ellos de forma subconsciente, sin que sea necesario anticipar cómo reaccionarán ante los mismos.

Obviamente, como se comenta en otros artículos de este monográfico, ambas características tienen una serie de problemas, que podríamos resumir en problemas éticos y problemas de efectividad. Desde le punto de vista de la ética, asumir que las administraciones públicas saben mejor que los ciudadanos qué es lo mejor para la sociedad en su conjunto plantea un debate moral profundo sobre los cimientos de nuestro sistema democrático. Desde el punto de vista de la efectividad, la pregunta no es ya tanto si se deben utilizar los nudges, sino si éstos funcionan y si son la herramienta más eficiente en términos de coste-beneficio en cada caso para conseguir los objetivos que se pretenden.

El propósito del artículo es realizar un análisis crítico del uso de los nudges como instrumento de las política pública, destacando su potencial en ciertos contextos pero también advirtiendo de los peligros asociados y comparándolo con otros instrumentos de política pública que pueden resultar complementarios o sustitutivos. El artículo está estructurado de la siguiente forma. La sección 2 realiza una comparación de los nudges con otros instrumentos inspirados por la Economía del Comportamiento utilizados para el diseño de políticas públicas. La sección 3 explica la evolución y la reciente aceptación de los nudges como elemento de las políticas públicas. La sección 4 contiene un comentario crítico sobre las ventajas y desventajas del uso de nudges. Por último, la sección 5 concluye con algunas recomendaciones para el uso de los nudges en el futuro.

\section{INTERVENCIONES BASADAS EN LA ECONOMÍA DEL COMPORTAMIENTO: NUDGES VS INCENTIVOS}

Podemos utilizar el modelo de Bemelmans-Videc y otros (1998) para intentar clasificar a los nudges como instrumento a disposición del gestor público. Esta tipología clasifica a las políticas públicas como palos, zanahorias o sermones. Los palos son herramientas reguladoras que buscan forzar el comportamiento de los ciudadanos. Las zanahorias proveen de incentivos para seducir a los individuos, mientras que los sermones, buscan persuadirles. Clasificar a los nudges en uno de estos tres grupos no es tarea sencilla. Los nudges claramente no son palos, puesto que no restringen las opciones disponibles para el individuo ni le castigan si no se comporta como el regulador pretende. Tampoco son zanahorias, puesto que buscan guiar al ciudadano de una forma inconsciente que no les haga sopesar los pros y contras de sus acciones, sin cambiar sus incentivos. Aún así, comparten con las zanahorias el hecho de que permiten la libertad de elección. Por último, los nudges tampoco son sermones, pues no pretenden persuadir a los ciudadanos de forma abierta mediante la provisión de información, aunque sí utilizan qué y como dan esa información para conseguir sus objetivos. Los nudges se encuentran en un término medio entre la zanahoria y el sermón, puesto que utilizan la información y la estructura física del entorno de decisión, lo que de forma un tanto rimbombante se ha llamado la arquitectura de la decisión, para influir, más que persuadir, en que el individuo decida libremente entre las opciones que se le presentan.

Se puede distinguir entre cuatro clases de intervenciones tipo nudge: 1) provisión de información, 2) cambios en el entorno físico, 3) cambios en las opciones ofrecidas «por defecto» y 4) el uso de las normas sociales y de la relevancia de las opciones ofrecidas (salience).

La provisión de información implica intervenciones que usen las referencias a otros comportamientos, el feedback, la forma de presentar o encuadrar la información (framing) o incluso la simplificación de mensajes, de manera que el individuo tome la decisión que político que ha diseñado una campaña informativa persigue. 
GAPP. Nueva Época - N. ${ }^{25}$, marzo 2021 - ISSN: 1989-8991 - DOI: https://doi.org/10.24965/gapp.i25.10864 - [Págs. 38-45] Número monográfico - Los nudges y el diseño conductual de políticas públicas

Mas allá de los nudges: Políticas públicas efectivas basadas en la evidencia de las ciencias del comportamiento

Los cambios en el entorno físico pueden suponer intervenciones que alteren el contexto de decisión, como por ejemplo el conocido ejemplo de cambiar el orden en el que se presentan las comidas de una cafetería estudiantil para intentar fomentar elecciones más sanas. Al situar alimentos más sanos al principio de un itinerario en el que hay que elegir distintas comidas, puede provocar que se elijan pronto los alimentos más sanos, provocando el que uno no se replantee su decisión y evite elegir comidas menos saludables.

En tercer lugar, los cambios en la opción «por defecto», implican elegir de forma consciente qué ocurre si el individuo no toma ninguna decisión, de forma que al no actuar ocurra lo que el gestor público pretende. El ejemplo clásico de opción por defecto es permitir que los ciudadanos elijan si quieren ser o no donantes de órganos al morir, pero en caso de que no hagan ningún trámite para expresar su preferencia, por defecto sean donantes. Esto podría explicar las enormes diferencias en la tasa efectiva de donación de órganos en países entre los que no existen grandes diferencias culturales, pero en los que sin embargo, la opción por defecto, el ser o no donante si no se toma ninguna decisión, puede llevar a que un alto porcentaje de individuos se comporte de acuerdo a lo establecido, sin incurrir en el mínimo coste administrativo que puede tener declararse o no donante de órganos.

Por último, el uso de normas sociales y de la relevancia de la información que se presenta implica utilizar información comparativa sobre lo que los demás están haciendo, de manera que, en ausencia de conocimiento sobre cómo uno debe comportarse en cierta situación, termine haciéndolo como indica la norma social o como se ha destacado que se comportan otros. Un ejemplo típico de indicación de norma social es informar a los contribuyentes de impuestos sobre el porcentaje de ciudadanos que en determinada fecha ya han pagado sus impuestos, de manera que el contribuyente que se ha retrasado en pagar, al tener mayor conocimiento sobre que otros ciudadanos ya han pagado, terminen haciéndolo.

Todos los ejemplos citados utilizan el conocimiento sobre la psicología de los individuos, para alterar su comportamiento de una manera no plenamente consciente, sino a través de un impulso. Aunque muchos de estos ejemplos son ampliamente conocidos y han resultado ser efectivos para inducir determinados comportamientos, queremos destacar que la profundización en el estudio sobre el proceso de toma de decisiones de los individuos y sobre su comportamiento que pretende la Economía del Comportamiento va mucho más allá del uso de los nudges. Por decirlo claro: ni todas las intervenciones behavioral son nudges, ni los nudges son necesariamente el instrumento más eficaz y duradero en todas las circunstancias para alcanzar los objetivos que persigue una política pública.

Como indican Lobel y Amir (2008) en su crítica a dos de los libros clásicos de la Economía del Comportamiento, Nudge de Thaler y Sunstein (2009) y Predicatbly Irrational de Ariely (2010), para evaluar la efectividad de un instrumento de política pública basado en la psicología y el comportamiento es fundamental comprender bien cuál es el mecanismo que causa que la decisión del individuo sea subóptima y, por tanto, si la intervención debería buscar eliminar la respuesta intuitiva (y quizá equivocada) de un individuo apelando de forma consciente a la mente racional o dejar que el poder de los procesos intuitivos cambien el comportamiento sin involucrarla. Su argumento se basa en la teoría de Kahneman que clasifica los procesos de decisión (de manera simplificada) en aquellos que utilizan la parte impulsiva de nuestra mente (sistema l) y aquellos más lentos y deliberados que implican sopesar de forma consciente las consecuencias de las distintas alternativas (sistema II). Esta forma de clasificar los procesos de decisión, instinto frente a racionalidad, es quizá un poco burda, puesto que ignora la posible existencia de un continuo entre lo puramente intuitivo y lo justificado, pero es útil para a su vez categorizar los distintos tipos de intervenciones psicológicas que se pueden utilizar para influir en el comportamiento de los ciudadanos. Por una parte, si creemos que el comportamiento inicialmente no deseado de los individuos se debe fundamentalmente a que están tomando una decisión intuitiva y no meditada, utilizando el sistema I, lo más directo puede ser utilizar un nudge, que cambie ese comportamiento instintivo inmediato. Por contra, un conocimiento profundo de las motivaciones de los individuos en decisiones más sopesadas que usan el sistema II, puede llevarnos al diseño de un mecanismo de incentivos, que obviamente no necesitan ser únicamente monetarias, sino que pueden tener en cuenta el amplio espectro de motivaciones humanas, proveyendo de razones conscientes adicionales para comportarse en la dirección deseada por el diseñador de la medida.

En muchas ocasiones, el arquitecto de la decisión tiene a su disposición ambos tipos de instrumentos, nudges e incentivos, para decidir cuál es mejor, tanto al ser utilizados por separado como combinándolos. La valoración de un tipo u otro de instrumento debe basarse no sólo en la comprensión de las razones que llevan al comportamiento subóptimo, sino también en un análisis coste-beneficio que incluya la efectividad y durabilidad de ambos tipos de medidas, el coste de implementarlas, las implicaciones éticas e incluso los posibles efectos adversos no previstos. 
GAPP. Nueva Época - N. ${ }^{\circ}$ 25, marzo 2021 - ISSN: 1989-8991 - DOI: https://doi.org/10.24965/gapp.i25.10864 - [Págs. 38-45] Número monográfico - Los nudges y el diseño conductual de políticas públicas

Mas allá de los nudges: Políticas públicas efectivas basadas en la evidencia de las ciencias del comportamiento

Antonio Cabrales / Pedro Rey Biel

Veamos con más detalle el ejemplo ya mencionado de convertir la donación de órganos en la opción por defecto para conseguir que efectivamente haya más órganos disponibles para los pacientes que los necesitan. Los defensores de este nudge argumentan que el simple cambio de la opción por defecto, pasando de una situación en la que los individuos tienen que declararse activamente donantes para serlo (opt-in) a otra en la que lo son de facto y deben hacer un trámite poco costoso para dejar de serlo (opt-out), conseguirá directamente aumentar las tasas de donación. Por el contrario, aquellos que creen que la decisión de ser donante de órganos es algo que nos importa lo suficiente como para que se trate de una decisión consciente en la que tenemos en cuenta múltiples factores, advierten del peligro de utilizar un nudge como la opción por defecto. Su preocupación viene motivada porque el mero hecho de cambiar la opción por defecto podría llevar a los individuos a considerar las opciones de forma más minuciosa, posiblemente creando una reacción adversa contra la donación. En el fondo, y dejando a una lado la discusión moral sobre la libertad de elección, el debate se centra aquí en la efectividad del uso de un nudge en un entorno concreto, y los posibles efectos contrarios que puede provocar. La efectividad de la medida dependerá por tanto de si tiene el impacto deseado y en la dirección deseada, y de si existen alternativas capaces de conseguir el mismo efecto sin quizá influir tanto en la libertad de elección y a un coste menor.

\section{EL AUGE DE LOS NUDGES COMO INTRUMENTO DE POLÍTICA PÚBLICA}

El argumento fundamental para el reciente auge del enfoque Nudge en el diseño de políticas públicas parte de que las autoridades a menudo tienen que guiar las acciones de los ciudadanos (o las empresas) y de los sesgos conductuales que poseemos los ciudadanos. Existen dos sesgos que resultan particularmente importantes para los defensores del enfoque Nudge. Uno es que, en ausencia de incentivos muy potentes, tendemos a guiarnos por el estatus quo. La última decisión tomada es la que vamos a mantener. El otro es un sesgo de «disponibilidad». Si hay una opción «destacada», vamos a tomarla. Las observaciones conductuales sugieren que no hay una opción «neutral». Si la autoridad no privilegia una opción, el individuo repetirá lo que venía haciendo. Si la autoridad consigue de alguna manera destacar una alternativa, mucha gente la tomará. En estas circunstancias la autoridad, tiene el deber de diseñar la «arquitectura de decisión», el menú de opciones disponibles, de manera que maximice el «bienestar real» del decisor, sin limitar de manera efectiva las opciones que éste puede tener. A esta forma de actuar se le conoce como paternalismo libertario.

Una gran virtud del paternalismo libertario es que ocupa un punto intermedio entre dos maneras de enfocar las políticas públicas. Una es el dirigismo de la izquierda tradicional o del fordismo clásico («puede comprar un modelo T del color que quiera... siempre que sea negro»). La otra es el laissez faire clásico, que supone que un gobierno que no interviene en absoluto preserva realmente la libertad del individuo y, si acaso, la misión del gobierno es aumentar el abanico de opciones de los consumidores, porque «el mercado es la mejor solución a cualquier problema». Los nudges rebaten diciendo que el dirigismo tradicional no tiene en cuenta la diversidad del ser humano, y que no todo el mundo, o no siempre, cae en los sesgos cognitivos que dan base a sus prescripciones. $Y$ a los libertarios tradicionales les recuerda que no hay tal cosa como las «preferencias inviolables» del individuo. Si vamos a elegir A si ésta es la opción privilegiada, o B si cambiamos de opinión, ¿cuál es realmente la preferencia «inviolable» que tiene el individuo? ¿cómo puede ser tan volátil e influenciable?. El paternalismo libertario se da cuenta de que la relativa facilidad con la que se puede influir en la decisión entre opciones implica el que no exista una manera neutral de presentar distintas opciones y, por tanto, dado que la autoridad debe presentar diversas posibilidades de todas formas, es mejor intentar decidir según lo que la sociedad acepte (o en su efecto la autoridad decida) qué es mejor para el individuo (o la sociedad).

Existe una segunda razón más pragmática para el auge los nudges. Cuando funcionan, los nudges producen resultados positivos a un coste muy bajo, o incluso sin coste. Pongamos por ejemplo los cambios en la carta que se enviaba a los contribuyentes británicos que se retrasaban en pagar sus impuestos. Modificar el texto de esa carta, añadiendo una frase que creara presión social (el $X \%$ de los contribuyentes han pagado ya sus impuestos) no tuvo apenas coste, pero consiguió elevar significativamente la recaudación del fisco y ayudó a la creación y consolidación de la primera agencia pública que usaba conceptos de Economía del Comportamiento para sugerir políticas, el Behavioural Insights Team (BIT), creado en 2010.

El libro de Sunstein y Thaler se publica en 2009, al principio de la "gran recesión» y del acceso al poder de David Cameron. El primer ministro se encuentra con un país en quiebra y con la necesidad de demostrar 
GAPP. Nueva Época - N. ${ }^{\circ}$ 25, marzo 2021 - ISSN: 1989-8991 - DOI: https://doi.org/10.24965/gapp.i25.10864 - [Págs. 38-45] Número monográfico - Los nudges y el diseño conductual de políticas públicas

Mas allá de los nudges: Políticas públicas efectivas basadas en la evidencia de las ciencias del comportamiento Antonio Cabrales / Pedro Rey Biel

que su gobierno podría conseguir resultados para sus ciudadanos. ¿Qué mejor que dar poder a un grupo de economistas y psicólogos para que consigan mejoras efectivas a coste cero? Mientras, al mismo tiempo, se hacían recortes en educación, o en el sistema nacional de salud (NHS). El Behavioural Insights Team (BIT) tuvo numerosos éxitos en sus primeros años, y consiguió una publicidad suficiente como para que en un momento dado se decidiera convertirlo en una empresa sin ánimo de lucro que vendía sus servicios, al sector público y privado. EI BIT, o como se le conoce más popularmente, el «Nudge Unit», emplea expertos varios campos relacionados con el espectro socio-económico, desde el desempleo y la recaudación de impuestos hasta médicos especialistas en salud mental.

En los últimos años, el BIT ha expandido su alcance a otros países como Australia o estados Unidos. En 2009, la administración Obama nombró a Cass Sunstein director de su Oficina de Información y Asuntos Regulatorias (OIRA, por sus siglas en inglés) y más tarde han creado su propia Nudge Unit. Agencias similares fueron creadas en Dinamarca (INudge You). De acuerdo al informe "Nudging All Over the World" (2014) del Economic and Social Research Council (ESRC), hasta en 136 países, incluidos Francia o Holanda han utilizado las ciencias del comportamiento para diseñar iniciativas de políticas sociales.

\section{RESERVAS AL USO DE LOS NUDGES}

Visto el argumentario científico, ético y pragmático que el enfoque Nudge ha tenido, y el relativo éxito de su expansión en diversos países, aunque no en España, parecería que el caso está cerrado y deberíamos abogar por la creación de una agencia similar en nuestro país. Nuestra perspectiva es un poco más compleja: aunque creemos que España se beneficiaría de tener una agencia con enfoqué científico que utilizara las ciencias del comportamiento, dicha agencia debería ser más ambiciosa, y no limitarse únicamente al enfoque nudge. A continuación, discutimos algunas de nuestras reservas frente al uso indiscriminado de los nudges.

Empecemos con la ciencia. Un problema de la aproximación Nudge es que no es una teoría completa del comportamiento humano. Como hemos visto, hay una serie de errores de decisión predecibles, que se pueden sistematizar vagamente con la idea de los dos sistemas de procesamiento, lento y rápido, pero en cada situación concreta no hay una serie de principios que ayuden a predecir qué va a pasar y cómo modificar el comportamiento. Esto contrasta fuertemente con la aproximación tradicional en economía, que, para problemas de decisión pura, suele sugerir una decisión única, si se conocen las preferencias y restricciones del decisor, y suele sugerir intervenciones muy concretas para modificar el comportamiento.

Esta superioridad es tan clara y útil que el propio Thaler recomendó en su conferencia presidencial de la American Economic Association de $2016^{2}$ simplemente adaptar el modelo estándar económico para incorporar los nuevos descubrimientos. Pero quizá no sea tan fácil hacerlo, puesto que muchos de esos descubrimientos tienen más de cuarenta años y aún no han sido integrados. Nos tememos que nos puede pasar como con la gran teoría unificada de física, cuya necesitada es clara desde hace un siglo, y ahí seguimos, con la relatividad y la teoría cuántica cada una por su lado.

Otro problema serio es la propia evidencia, que puede no ser tan sólida como algunas de las anécdotas sobre nudges más conocidas y llamativas parecen sugerir. El éxito de estas medidas depende de mil detalles contextuales. Hemos descubierto en tiempo recientes que todas las ciencias sufren un problema de replicabilidad de sus resultados empíricos. Pero la psicología ha sido particularmente afectada por este problema. Decenas de experimentos famosos han ido siendo puestos en cuestión uno detrás de otro. Leer libros de economía conductual hoy, conociendo la evidencia a fondo, nos lleva a ser mucho más prudentes. Nos parece indudable que hay un sesgo de publicación sobre resultados «sorprendentes» que parecen desmontar «el mito de la racionalidad humana». Esos sesgos existen, pero su efecto es heterogéneo en la población, muy dependiente de contextos que aún no entendemos bien y con un poder limitado.

Esto es importante porque incluso las intervenciones que funcionan de verdad podrían tener una duración reducida, algo que, por ejemplo en áreas como la educación, sabemos que es un problema serio. Y podrían ser afectados por el paso del tiempo. Por poner un ejemplo, una intervención estándar tipo Nudge como los recordatorios para realizar una acción indeseada (por ejemplo, por aburrida), puede funcionar en ocasiones pero, por lo general, su efecto es muy limitado en el tiempo una vez pierden su carácter de novedad.

2 Resumida aquí: https://nadaesgratis.es/pedro-rey-biel/de-mr-spock-a-homer-simpson-notas-presidenciales-sobre-la-economia-del-comportamiento (22-1-2016). 
GAPP. Nueva Época - N. ${ }^{\circ}$ 25, marzo 2021 - ISSN: 1989-8991 - DOI: https://doi.org/10.24965/gapp.i25.10864 - [Págs. 38-45] Número monográfico - Los nudges y el diseño conductual de políticas públicas

Mas allá de los nudges: Políticas públicas efectivas basadas en la evidencia de las ciencias del comportamiento

Además, los argumentos pragmáticos y políticos en favor del enfoque Nudge tienen también problemas. Recuerden que el éxito de la aproximación nace en parte de que las intervenciones resultan (casi) «gratis». Ésto tiene dos consecuencias negativas. La más inmediata es que el BIT se usó por el gobierno británico en parte para «blanquear» los recortes presupuestarios en muchas políticas sociales y no hacer suficientes esfuerzos en atacar realmente las raíces profundas de importantes problemas sociales. El impacto más serio viene de que acostumbrarse a resultados gratis puede hacernos perder la perspectiva de que la mayor parte de intervenciones para cambiar la conducta no serán gratis. Quizá sea exagerado decir que «nada es gratis», pero hay mucha sabiduría en la frase popular de que «al que algo quiere, algo le cuesta». Podemos usar las «opciones por defecto» para mejorar las tasas de ahorro de la población, pero si hay un motivo económico legítimo para intervenir en la tasa de ahorro, no va a quedar más remedio que penalizar el consumo presente, o bonificar el consumo futuro.

También caben dudas sobre el supuesto de benevolencia que subyace a la idea de que el gobierno elija la opción por defecto o diseñe la arquitectura de elección. Con gran probabilidad un político podría utilizar estos instrumentos para su propio beneficiarse de manera completamente transparente y sin tapujos. Esto no es sólo una posibilidad teórica y es una preocupación latente en muchas de las reticencias que caricaturizan el enfoque nudge como una mera manipulación. Las empresas, y en particular los expertos en marketing, llevan siglos utilizando la arquitectura de la decisión para influenciar nuestras compras y maximizar su beneficio. ¿O se creen que la posición de los productos en los lineales del supermercado, o su proximidad a las cajas lo decide el azar, o la conveniencia del consumidor? Es verdad que ésto no es un juego de suma cero, y el consumidor puede ganar con ello, pero si hay posibilidad de aumentar el beneficio a costa de su propia ingenuidad, no dude de que lo harán. Y si las empresas lo hacen, ¿por qué no lo va a hacer el gobierno?

Por todo lo anterior, en un sentido bien definido, el enfoque nudge no es nuevo, no es correcto y es incompleto. Antes de crear BITs por todas partes, es importante diseñarlo bien. Por ejemplo, una debilidad del BIT británico es que era una oficina dependiente del primer ministro, y por tanto no tenía obligación de ofrecer consejos independientes. Esto ha llevado, por ejemplo y en el contexto actual de la pandemia del coronavirus, a embarazosas declaraciones de David Halpern, su presidente y a la percepción de que el fallido intento del gobierno británico de no confinar a su población estaba basada en mala ciencia conductual ${ }^{3}$. Es muy probable que un órgano más independiente hubiera tomado mayor distancia respecto a estas malas decisiones.

También es importante que el mandato de una unidad de ciencia conductual asociada al gobierno NO tenga como misión gastar poco dinero, sino gastarlo bien (como cualquier otra unidad del gobierno, por otra parte).

Desde el punto de vista científico nos parece que hay dos cosas muy importantes. La primera es prestar menos atención a los resultados «llamativos», y mayor a la replicabilidad de los resultados. Para lo cual la nueva tendencia al pre-rregistro de experimentos y otras innovaciones para impedir la práctica de manipular los datos hasta que los resultados sobrepasen el umbral de error estadístico requerido, conocido como «p-hacking», son muy bienvenidas. La segunda, a la que ya hemos aludido, es acercarnos a la gran teoría unificada del comportamiento humano, que tome en cuenta la evidencia real, pero que no se conforme con hilar anécdotas o hacer cambios marginales a los modelos estándar.

\section{CONCLUSIONES Y RECOMENDACIONES}

La cada vez mayor aceptación de la evidencia empírica proveniente de las ciencias del comportamiento por parte de los responsables públicos, es una muy buena noticia, puesto que supone uno de los muchos pasos que necesitamos dar hacia el diseño y evaluación de políticas basadas en la evidencia sobre lo que realmente mejora nuestras sociedades. Esta afirmación no implica desterrar la ideología de la decisión pública pero ésta debe ceñirse más a la elección entre objetivos, y no confundir la discusión sobre qué es lo que funciona para conseguirlos, lo que puede esclarecerse de forma mucho más precisa a la vista de los datos.

La creación de agencias gubernamentales que utilicen las ciencias del comportamiento para conseguir de una manera eficaz sus objetivos es uno de los requisitos imprescindibles en la modernización de las políticas públicas. Requisito además que va de la mano de otros de los pasos necesarios del proceso de

3 https://www.ncbi.nlm.nih.gov/pmc/articles/PMC7201134/ y https://scramnews.com/who-is-david-halpern-government-response-nudge-unit-coronavirus/. 
GAPP. Nueva Época - N. ${ }^{\circ}$ 25, marzo 2021 - ISSN: 1989-8991 - DOI: https://doi.org/10.24965/gapp.i25.10864 - [Págs. 38-45] Número monográfico - Los nudges y el diseño conductual de políticas públicas

Mas allá de los nudges: Políticas públicas efectivas basadas en la evidencia de las ciencias del comportamiento

Antonio Cabrales / Pedro Rey Biel

modernización: evaluación independiente y rigurosa de las políticas públicas, disponibilidad pública de datos de los diferentes niveles de la administración, diseño de intervenciones públicas de forma que permitan realmente ser evaluadas y una actitud favorable a la experimentación, a probar qué es lo que funciona y porqué, antes de implementar nuevas políticas. El enfoque de economía del comportamiento ha mostrado éxitos en todas estas tareas pendientes, y por ello no debemos ceñirnos sólo al uso de nudges, por muy atractivos que resulten debido a algunos casos de éxito y a su bajo coste.

\section{REFERENCIAS BIBLIOGRÁFICAS}

Ariely, D. (2010). Predictably Irrational: The Hidden Forces That Shape Our Decisions. Harper Perennial.

Bemelmans-Videc, M. L., Rist, C. y Vedung, E. (1998). Carrots, ticks and Sermons: Policy Instruments and their evaluation. Transaction Publishers.

Bentham, J. (1789, reeditado 2008). Los principios de la moral y la legislación. Claridad.

Howell, R., Jones, R., Lilley R., Pykett, J. y Whitehead, M. (septiembre de 2014). Nudging All Over the World. Assesing the Global Impact of the Behavioural Sciences on Public Policy. Economic and Social Research Council (ESRC). https://changingbehaviours.files.wordpress.com/2014/09/nudgedesignfinal.pdf

Lobel, O., Amir, O. (2008). Stumble, Nudge, Predict: How Behavioral Economics Informs Law and Policy. Columbia Law Review, 108(8), 2.098-2.138.

Kahneman, D., Slovic, P., y Tversky, A. (1982). Judgment Under Uncertainty: Heuristics And Biases. Cambridge University Press. https://doi.org/10.1017/CBO9780511809477

Smith, A. (1759). The Theory Of Moral Sentiments. Impreso por A. Millar, A. Kincaid y J. Bell, en Edimburgo.

Thaler, R. H., y Sunstein, C. R. (2009). Nudge: Improving Decisions About Health, Wealth, And Happiness (edición revisada y ampliada). Penguin Books. 